\title{
CLIPPERS: A case report with radiology, three serial biopsies and a literature review
}

Turnquist $\mathrm{C}^{1}$, Pretorius $\mathrm{PM}^{2}$, DeLuca $\mathrm{GC}^{3}$, Halliday $\mathrm{J}^{4}$, Kerr $\mathrm{R}^{4}$, Hofer $\mathrm{M}^{5}$

1. University of Oxford Medical School, John Radcliffe Hospital, Oxford, UK

2. Department of Neuroradiology, Oxford University Hospitals NHS Foundation Trust, Oxford, UK

3. Nuffield Department of Clinical Neurosciences, John Radcliffe Hospital, Oxford, UK

4. Department of Neurosurgery, Oxford University Hospitals NHS Foundation Trust, Oxford, UK

5. Department of Neuropathology, Oxford University Hospitals NHS Foundation Trust, Oxford, UK

Correspondence: casmir.turnquist@medschool.ox.ac.uk; University of Oxford Medical School, John Radcliffe Hospital, Oxford, OX3 9DU, UK

\section{Abstract}

20 Chronic Lymphocytic Inflammation with Pontine Perivascular Enhancement Responsive

21 to Steroids (CLIPPERS) is a rare central nervous system inflammatory disorder

22 primarily affecting the brainstem and cerebellum. We report a case of CLIPPERS in a

23 45-year-old man presenting with left facial numbness and dizziness. Imaging studies

24 were conducted repeatedly over an 8-year follow up period. Given diagnostic

25 uncertainty in the early stages of the disease, three serial biopsies were obtained, which

26 together with the clinical and radiological findings led to the diagnosis. This case

27 highlights the diagnostic challenges regarding the rare entity of CLIPPERS and

28 discusses the main differential diagnoses that are necessary to consider. Additionally,

29 some of the atypical features of this case, including the presenting finding of a large,

30 solidly enhancing lesion on radiological imaging and prominent plasma cells on

31 pathology, contribute expanding the spectrum of appearances for CLIPPERS.

32 Key words: CLIPPERS-neuroinflammation-lymphocytic infiltrate-brainstem-cerebellum 


\section{Introduction}

2 Chronic Lymphocytic Inflammation with Pontine Perivascular Enhancement Responsive

3 to Steroids (CLIPPERS) is an inflammatory disease predominantly affecting the

4 brainstem and cerebellum with distinct clinical, radiological and histopathological

5 features. CLIPPERS was first described by Pittock et al in 2010 as a relapsing-remitting

6 chronic inflammatory disorder of the central nervous system [1]. The mean age is

7 around the fifth decade with a slight male predominance [2].

9 Clinically, patients present with brainstem and cerebellar symptoms and signs including

10 gait ataxia, diplopia, and dysarthria. Less common clinical features include dizziness,

11 tinnitus, tremor, nystagmus, spasticity and sensory loss. The presence of systemic

12 symptoms such as weight loss and fever are atypical and should prompt investigation of

13 other causes. Gadolinium enhancement MRI reveals characteristic multiple punctate-

14 enhancing lesions or 'peppering' that is typically confined to the pons, but may also

15 extend to adjacent central nervous system (CNS) structures including the cerebellar

16 peduncles, cerebellar hemispheres, and spinal cord [1]-[4]. Most lesions are $<3 \mathrm{~mm}$ in

17 diameter and are symmetrical. Lesions typically become less numerous and smaller as

18 the distance from the pons increases. No oedema, mass effect, nor vasculitic changes

19 are present. Both the clinical symptoms and signs and the radiological findings are

20 exquisitely responsive to steroids.

22 In terms of the neuropathological findings, the lesions in CLIPPERS are characterised

23 by a pronounced angiocentric and parenchymal lymphocytic infiltrate composed 
1 predominantly of CD3-positive T-lymphocytes in white and grey matter, a smaller

2 number of CD68-positive histiocytes and CD20-expressing B-lymphocytes, with

3 activated microglia [1]. There are no Langerhans-type giant cells nor granulomas and

4 myelin is intact. Immunostaining for fungi, toxoplasma, CMV, EBV and JC virus is

5 negative. As no pathological biomarker exists, it is important to rule out alternative

6 diagnoses, notably, granulomatous diseases, lymphoma, infection, and vasculitis.

8 We describe a patient who presented with left facial numbness and dizziness and an

9 MRI showing an enhancing lesion in the left side of the pons / left middle cerebellar

10 peduncle. Histopathological findings from three biopsies were supportive of CLIPPERS.

11 The patient has been followed-up for the past 8 years with good clinical outcome on

12 long-term immunosuppression.

\section{Materials and methods}

15 Magnetic resonance imaging (MRI) studies were conducted over an 8-year follow up of

16 this patient and reviewed by a neuroradiologist (PMP) at the Department of

17 Neuroradiology, Oxford University Hospitals NHS Foundation Trust. Selected axial T1-

18 weighted gadolinium-enhanced images at the level of the mid pons and middle

19 cerebellar peduncles, which led to the diagnosis are described and included in this case

20 report. 
1 Histology and immunohistochemical $(\mathrm{IHC})$ evaluation was performed at the Department

2 of Neuropathology, Oxford University Hospitals NHS Foundation Trust. Four-micron

3 paraffin-embedded tissue sections were deparaffinised, rehydrated and incubated with

4 antibodies including CD2, CD3, CD4, CD5, CD7, CD8, CD10, CD20, CD79a, Tdt and

5 MIB-1. Immunostaining was also performed for toxoplasma, CMV and JC virus. A list of

6 antibodies, dilutions and suppliers is listed in Table 1. Toxoplasma immunostaining was

7 performed courtesy of Professor David Ferguson (formerly at the Nuffield Department of

8 Clinical Laboratory Science, University of Oxford). Sections were double labelled

9 with Toxoplasma stage-specific antibodies (tachyzoite-specific rabbit anti-SAG1 raised

10 to the recombinant protein [5] and bradyzoite-specific mouse anti-BAG1 raised to the

11 recombinant protein [6]) and visualized using goat anti-rabbit immunoglobulin

12 conjugated to fluorescein isothiocyanate and goat anti-mouse immunoglobulin

13 conjugated to Texas red and counterstained with 4',6-diamidino-2-phenylindole.

14 Additionally images from the 2 most representative biopsies demonstrating H\&E

15 staining and CD3, and CD79a immunostaining are included in the report.

16 Immunoperoxidase visualization and antigen retrieval was performed following the

17 manufacturer's instructions. Images were captured using an Olympus camera SC100

18 camera and cellSens Standard software.

20 EBV in situ hybridisation was performed using the Leica Bond EBER Probe (catalogue

21 number: PB0589) and stain using the Bond III ISH protocol A, as per manufacturer's

22 instructions. 
1 Clonality studies were performed in Molecular Haematology, Oxford Genetic Laboratory

2 using the polymerase chain reaction (PCR)-based IdentiClone Gene Clonality Assays

3 (Invivoscribe Technologies, Inc.), which are EuroClonality validated multiplex protocols.

5 Case report

6 A 45-year-old white British male presented in May 2010 with a tingling sensation along

7 the medial end of the left side of his jaw and neck. He was also noted to veer to the left

8 side on assessment of his gait. Over the next few weeks he developed dizziness and

9 early morning nausea and vomiting without headaches. An MRI with gadolinium (Figure

10 1) revealed a left cerebellar solidly-enhancing lesion with oedema. He was started on

11 dexamethasone after which his symptoms resolved. An initial biopsy (Figure 2) was

12 obtained of the left middle cerebellar peduncle, which revealed heavy perivascular and

13 parenchymal infiltration by small to medium sized T-cells that were CD2, CD3, CD4,

14 CD5, and CD7-positive but CD10 and CD8-negative on immunohistochemistry. A small

15 subset of cells expressed B- and plasma cell markers, CD20 and CD79a. MIB-1

16 staining revealed a low proliferative rate of $<1 \%$. PCR investigating T-cell receptor

17 rearrangement revealed no evidence of clonality. Additionally, PCR studies investigating

18 clonal rearrangements of the B-cell receptor were performed, but the results were

19 inconclusive due to insufficient material. Further immunostaining for fungi, toxoplasma,

20 CMV, EBV and JC virus was negative. There were no Langerhans-type giant cells nor

21 granulomas and myelin was intact. An MRI in July 2010 showed that the lesion had

22 diminished in response to steroids. The steroid-responsiveness along with the solid 
1 nature of the lesion raised suspicion of a diagnosis of treated lymphoma of T- or B-cell

2 origin at the time.

4 In April 2011, while the patient was off of steroids, a repeat MRI brain scan revealed 5 punctate enhancing lesions within the pons and left middle cerebellar peduncle, which 6 prompted a second biopsy in May 2011 while the patient was off steroids. Pathology

7 (Figure 3) revealed more B-cells with perivascular inflammatory aggregates including 8 lymphoid cells of varying sizes, plasma cells, and foamy macrophages. Rare atypical

9 cells with irregular chromatin and multiple nucleoli were identified in addition to at least

10 three mitotic figures within the aggregates. The MIB-1 index was approximately $10 \%$.

11 Further immunostaining for EBER in situ hybridisation and SV40 were negative.

13 In August 2011, a third biopsy was taken (not shown) which was similar to the second 14 biopsy in terms of the cell populations identified and the MIB-1 index. Importantly, there

15 was no evidence of lymphoma, which increased the likelihood of an immune-mediated

16 inflammatory process. Taken together, the marked steroid responsiveness, the evolving

17 MRI imaging features along with the consistent lack of pathological evidence for

18 lymphoma strongly supported a CLIPPERS diagnosis. The patient has been followed up

19 for the past 8 years and has had a good outcome with long term immunosuppression.

\section{Discussion}

22 The case presented here possesses several unusual features which contribute to the 23 expanding spectrum of appearances of CLIPPERS. They include radiological findings of 
1 a large, solitary and asymmetrical lesion at presentation along with the prominence of

2 plasma cells on neuropathological examination. While the imaging findings during

3 periods of relapse were more typical of CLIPPERS with multiple small enhancing foci.

4 However, the relatively large, solitary enhancing lesion with all lesions to the left of the

5 midline at presentation, has not previously been described in CLIPPERS to our

6 knowledge. Both the size of the lesion $>3 \mathrm{~mm}$ and its asymmetry are features that

7 would suggest a diagnosis of primary CNS lymphoma, but this case demonstrates that

8 these features can also exist in CLIPPERS. Additionally, plasma cells were fairly

9 prominent in this case compared to the majority of previous reports, in which they are

10 rare or absent [1], [2], [7]. Plasma cell infiltration has been reported in a few cases

11 although their significance remains unknown [8], [9]. Finally, this case highlights the

12 evolving pathological and radiological features of CLIPPERS that are evident through

13 serial biopsies and imaging in this case.

15 The aetiology of this rare disease remains poorly understood. However, several

16 hypotheses exist including an inflammatory aetiology, which is suggested by the

17 presence of CD4-positive T cells, response to immunosuppression, and a temporal

18 relation to vaccination [10] and to herpes zoster reactivation [11]. Other aetiological

19 possibilities include a pre-malignant state whereby lymphocytic infiltration precedes

20 primary CNS B-cell lymphoma as described in a few case reports originally diagnosed

21 as CLIPPERS [12]-[16]. Additionally, a vascular aetiology has been proposed due to

22 reports of both small [17] and large [18] vessel abnormalities and infarction [19], but 
1 vascular changes have not consistently been reported in the literature. Whilst the

2 aetiology of CLIPPERS remains uncertain, advances in immunopathology and radiology

3 have allowed CLIPPERS to be recognised as a distinct entity that differs considerably in

4 its clinical presentation, immunological characteristics, radiological findings and its 5 responsiveness to steroids.

7 Recently, diagnostic criteria were proposed for definite and probable CLIPPERS [8].

8 While definite CLIPPERS requires the fulfilment of all clinical, radiological, and

9 neuropathological criteria, probable CLIPPERS does not require neuropathological

10 findings. The neuropathological criteria include dense perivascular lymphocytic

11 inflammation, T-cell predominant infiltration (CD4 > CD8), absence of myelin loss and

12 lack of alternative diagnosis for pathological findings. The clinical criteria proposed

13 include subacute pontocerebellar dysfunction, response to corticosteroids, no peripheral

14 nervous system involvement, and lack of alternative diagnosis for clinical findings.

15 Finally, the radiological criteria include a homogenous, gadolinium-enhancing nodules

16 without ring enhancement measuring $<3 \mathrm{~mm}$ in diameter, improvement with

17 corticosteroids, homogenous T2 signal abnormality where the degree of T2 does not

18 exceed the size of the area of post-gadolinium enhancement, and spinal cord lesions

19 with similar T2 signal. The case presented here suggests that the disease spectrum of

20 CLIPPERS may be broader than outlined by the above criteria in terms of lesion size

21 and radiological findings that changes markedly over the disease course. 
1 As no definitive biomarker for CLIPPER exists, the formulation of pathologic diagnostic

2 criteria for CLIPPERS is essential to ensure alternative diagnoses are appropriately

3 considered. Diagnostic criteria also support research into CLIPPERS aetiology.

4 Additional external validation involving multiple centres may be required to confirm the 5 recently proposed diagnostic criteria for this entity.

\section{Acknowledgement}

8 The authors would like to thank the patient and his family for allowing us to write this

9 case. Additionally, we are grateful to Dr. James Morris for his comments to the

10 manuscript. We also thank Molecular Haematology, Oxford Genetic Laboratory for

11 performing the clonality studies and some of the immunohistochemistry as well as

12 Professor David Ferguson for performing the toxoplasma immunostaining. 
Conflict of interest

The authors declare no conflict of interest.

\section{References}

[1] S. J. Pittock et al., "Chronic lymphocytic inflammation with pontine perivascular enhancement responsive to steroids (CLIPPERS)," Brain, vol. 133, no. 9, pp. 26262634, Sep. 2010.

[2] A. Dudesek et al., "CLIPPERS: chronic lymphocytic inflammation with pontine perivascular enhancement responsive to steroids. Review of an increasingly recognized entity within the spectrum of inflammatory central nervous system disorders," Clin Exp Immunol, vol. 175, no. 3, pp. 385-396, Mar. 2014.

[3] A. K. Bag, J. J. Davenport, J. R. Hackney, R. Roy, and H. M. Fathallah-Shaykh, "Case 212: Chronic Lymphocytic Inflammation with Pontine Perivascular Enhancement Responsive to Steroids," Radiology, vol. 273, no. 3, pp. 940-947, Nov. 2014.

[4] W. Maenhoudt, K. Ramboer, and V. Maqueda, "A Rare Cause of Dizziness and Gait Ataxia: CLIPPERS Syndrome," Journal of the Belgian Society of Radiology, vol. 100, no. 1, Feb. 2016.

[5] D. Harning, J. Spenter, A. Metsis, J. Vuust, and E. Petersen, "Recombinant Toxoplasma gondii surface antigen 1 (P30) expressed in Escherichia coli is recognized by human Toxoplasma-specific immunoglobulin $\mathrm{M}(\lg M)$ and $\lg G$ antibodies.," Clin. Diagn. Lab. Immunol., vol. 3, no. 3, pp. 355-357, May 1996.

[6] W. Bohne, U. Gross, D. J. P. Ferguson, and J. Heesemann, "Cloning and characterization of a bradyzoite-specifically expressed gene (hsp30/bag1) of Toxoplasma gondii, related to genes encoding small heat-shock proteins of plants," Molecular Microbiology, vol. 16, no. 6, pp. 1221-1230, 1995.

[7] N. G. Simon et al., "Expanding the clinical, radiological and neuropathological phenotype of chronic lymphocytic inflammation with pontine perivascular enhancement responsive to steroids (CLIPPERS)," J Neurol Neurosurg Psychiatry, vol. 83, no. 1, pp. 15-22, Jan. 2012.

[8] W. O. Tobin et al., "Diagnostic criteria for chronic lymphocytic inflammation with pontine perivascular enhancement responsive to steroids (CLIPPERS)," Brain, vol. 140, no. 9, pp. 2415-2425, Sep. 2017.

[9] R. Rössling, D. Pehl, M. Lingnau, and H. Prüss, "A case of CLIPPERS challenging the new diagnostic criteria," Brain, vol. 141, no. 2, pp. e12-e12, Feb. 2018.

[10] P. B. Hillesheim, J. R. Parker, J. C. Parker, E. Escott, and J. R. Berger, "Chronic lymphocytic inflammation with pontine perivascular enhancement responsive to steroids following influenza vaccination," Arch. Pathol. Lab. Med., vol. 136, no. 6, pp. 681-685, Jun. 2012.

[11] L. Wang, E. A. Holthaus, X. F. Jimenez, J. Tavee, and Y. Li, "MRI evolution of CLIPPERS syndrome following herpes zoster infection," J. Neurol. Sci., vol. 348, no. 1-2, pp. 277-278, Jan. 2015. 
[12] G. Taieb, D. Renard, J. M. Joujoux, and P. Labauge, "Cutaneous lesions in a CLIPPERS patient: further confusion between CLIPPERS and grade I lymphomatoid granulomatosis," J. Cutan. Pathol., vol. 41, no. 9, pp. 759-760, Sep. 2014.

[13] N. Limousin et al., "Brain biopsy is required in steroid-resistant patients with chronic lymphocytic inflammation with pontine perivascular enhancement responsive to steroids (CLIPPERS)," J Neurooncol, vol. 107, no. 1, pp. 223-224, Mar. 2012.

[14] H. J. De Graaff, M. P. Wattjes, A. J. Rozemuller-Kwakkel, A. Petzold, and J. Killestein, "Fatal B-cell lymphoma following chronic lymphocytic inflammation with pontine perivascular enhancement responsive to steroids," JAMA Neurol, vol. 70, no. 7, pp. 915-918, Jul. 2013.

[15] K. Alsherbini, B. Beinlich, and M. S. Salamat, "Diffusely Infiltrating Central Nervous System Lymphoma Involving the Brainstem in an Immune-Competent Patient," JAMA Neurol, vol. 71, no. 1, pp. 110-111, Jan. 2014.

[16] A. W. Lin et al., "Emergence of Primary CNS Lymphoma in a Patient with Findings of CLIPPERS," Can J Neurol Sci, vol. 41, no. 4, pp. 528-529, Jul. 2014.

[17] B. K. Kleinschmidt-DeMasters and M. West, "CLIPPERS with chronic small vessel damage: more overlap with small vessel vasculitis?," J. Neuropathol. Exp. Neurol., vol. 73, no. 3, pp. 262-267, Mar. 2014.

[18] M. Buttmann, I. Metz, I. Brecht, W. Brück, and M. Warmuth-Metz, "Atypical chronic lymphocytic inflammation with pontocerebellar perivascular enhancement responsive to steroids (CLIPPERS), primary angiitis of the CNS mimicking CLIPPERS or overlap syndrome? A case report," J. Neurol. Sci., vol. 324, no. 1-2, pp. 183-186, Jan. 2013.

[19] G. Saigal and R. Quencer, "Acute lacunar infarcts in CLIPPERS: is the chronic infiltrative lymphocytic perivascular disease process to blame?," Neuroradiol J, vol. 26, no. 6, pp. 629-635, Dec. 2013. 
1 Figures and Tables

2

Figure 1
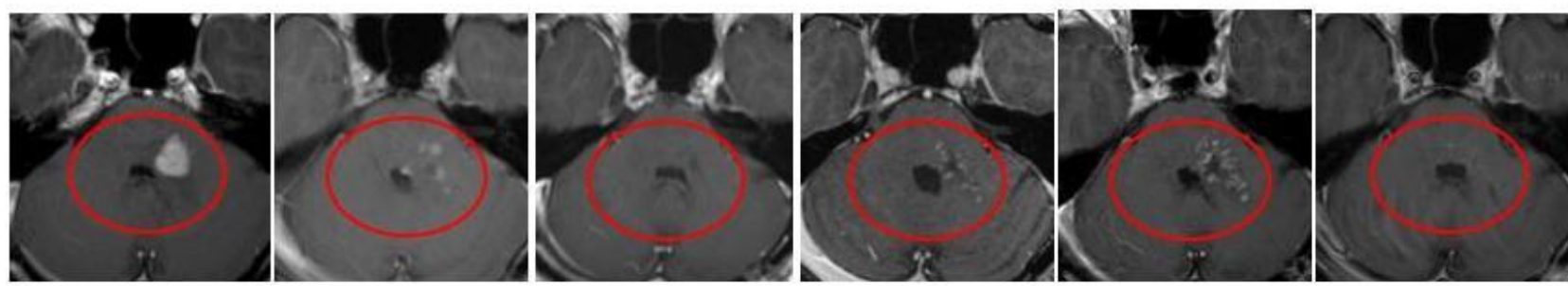

May 2010

Dec 2010

April 2011

May 2011

June 2011

3

Figure 2

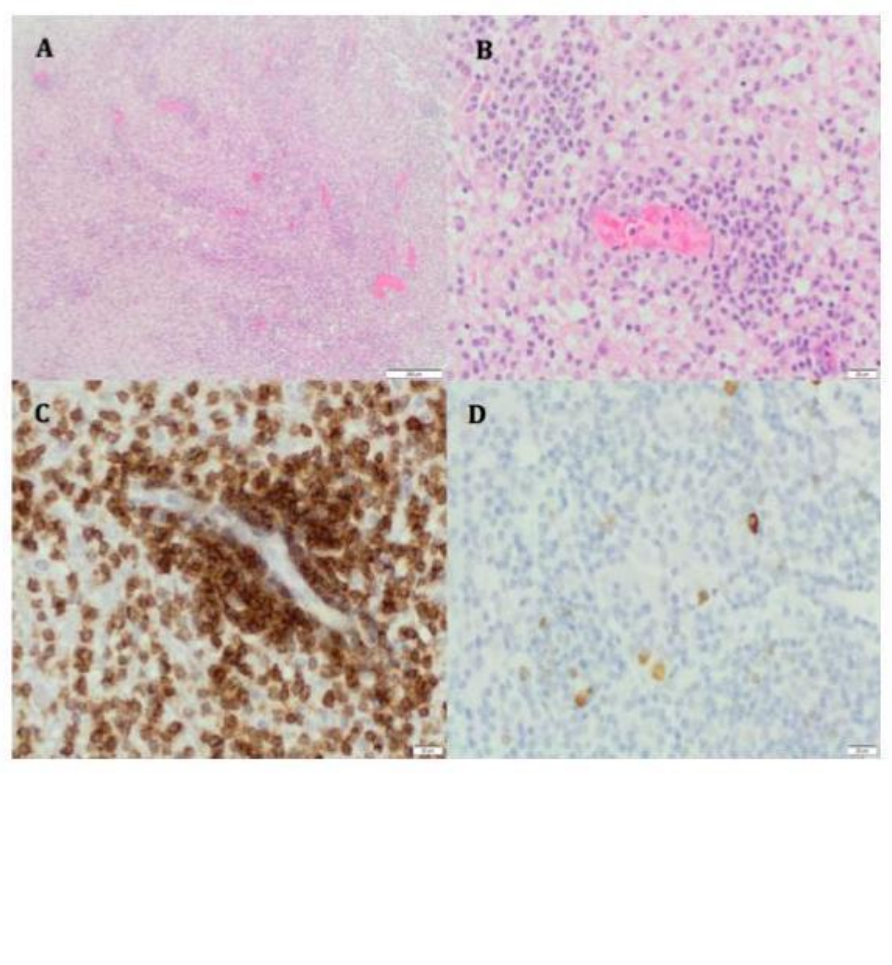




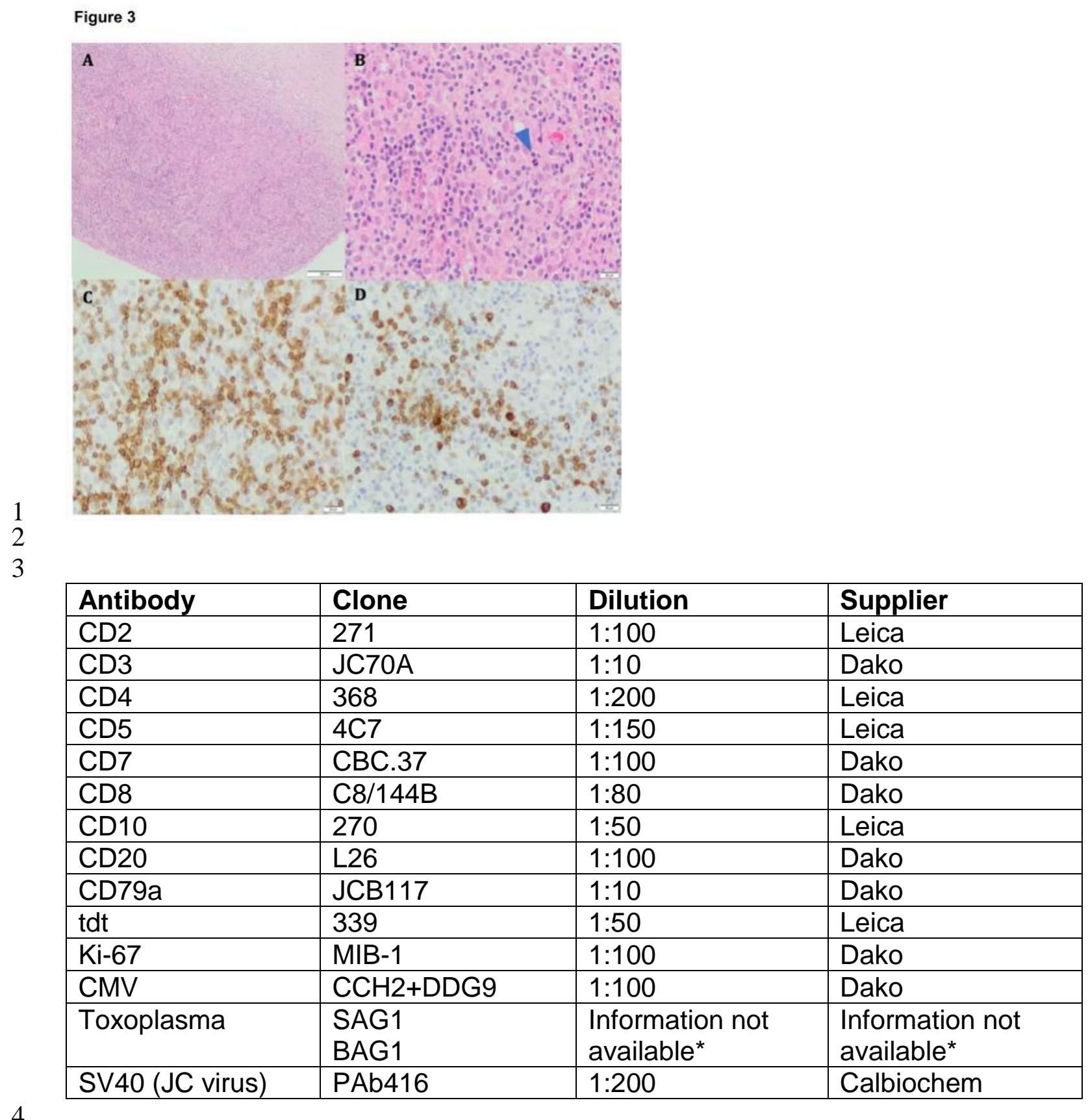


Figure and table legends

Figure 1. Selected axial T1-weighted gadolinium-enhanced images at the level of the mid pons and middle cerebellar peduncles. The series of images represent more than one year of the patient's history from the time of presentation and demonstrates the waxing and waning course of the imaging abnormalities in the pons and left middle cerebellar peduncle. White arrowheads on the timeline indicate when the biopsies were performed and red arrowheads indicate when steroids were given.

Figure 2. First biopsy (May 2010). Initially diagnosed as suspected T- or B-cell lymphoma. (A) H\&E stain showing small to medium sized lymphocytes with irregular non-cleaved round nuclei and finely dispersed chromatin and foamy macrophages; (B) $\mathrm{H} \& \mathrm{E}$ stain highlighting the perivascular distribution of lymphocytes; (C) CD3 staining of T-lymphocytes; (D) Sparse CD79a staining of B-lymphocytes and plasma cells.

Figure 3 - Second biopsy (May 2011). Diagnosis of immune related inflammatory process of unknown significance, suggestive of CLIPPERS. (A-B) H\&E stain showing similar findings to the first biopsy with small to mediums sized lymphocytes and foamy macrophages and sparse mitotic figures (arrowhead). (C) CD3 staining of Tlymphocytes; (D) CD79a staining of B-lymphocytes and plasma cells.

Table 1. Primary antibodies. *Dilutions and suppliers of these antibodies is not available as Professor David Ferguson, the expert in the field who conducted these experiments, is now retired. 